\title{
Serum adenosine deaminase and C-reactive protein in diabetic patients with and without neuropathy
}

\section{ABSTRACT}

Background. Diabetic Peripheral Neuropathy (DPN) is one major microvascular complication of long-standing diabetes mellitus with a prevalence of $50-60 \%$. DPN leads to decreased quality of life, increased morbidity, and mortality. $C$ reactive protein (CRP) and adenosine deaminase (ADA) are independent inflammatory markers; the current study aims to evaluate and compare serum ADA, CRP in diabetic patients with and without neuropathy.

Methods. A prospective study was done on 160 diabetic patients with and without neuropathy who visited the Medicine Department of Kasturba Hospital Manipal. Patients were grouped into two: diabetics with neuropathy $(n=80)$ and without neuropathy $(n=80)$. Serum samples were collected and analyzed for CRP and ADA.

Results. The data were analyzed using the independent t-test and Mann-Whitney $U$ test. There was a significantly high level of ADA $(P=0.032)$ in neuropathy patients when compared with the non-DPN group. No significant difference was seen in CRP levels $(P=0.123)$ in DPN patients compared to non-DPN patients.
Address for correspondence:

Anju Muraleedharan

Department of Medical Laboratory Technology,

Manipal College of Health Professions,

Manipal Academy of Higher Education.

Manipal, Karnataka, India, 576104

e-mail: anju.muraleedharan@manipal.edu

Clinical Diabetology 2021, 10; 5: 407-411

DOI: 10.5603/DK.a2021.0046

Received: 6.11.2020

Accepted: 8.03.2021
Conclusion. Monitoring of inflammatory markers like ADA in diabetic patients can provide insight into preventing DPN and its associated complications. (Clin Diabetol 2021; 10; 5: 407-411)

Key words: diabetes mellitus, diabetic peripheral neuropathy, C-reactive protein, adenosine deaminase

\section{Introduction}

Diabetes mellitus (DM) is a severe and long-term condition that has a significant impact on the lives and well-being of societies and families worldwide. In 2019 it was estimated to have caused 4.2 million deaths globally [1]. In India, about 463 million people were affected by diabetes in 2019, representing $9.3 \%$ of adults worldwide; by 2045 this will increase to 700 million [2]. DM is a chronic disorder that leads to hyperglycemia, metabolic abnormalities, and long-term macro- and microvascular complications involving the eyes, blood vessels, nerves, and kidneys [3]. Diabetic neuropathy is a common microvascular complication with the prevalence of $50-60 \%$ in T2DM individuals. Neuropathy leads to a decrease in nerve activities and nerve blood perfusion with persistent nerve damage. Diabetic peripheral polyneuropathy (DPP) can cause an increased possibility of foot ulceration and necrosis, which may lead to lower extremity amputation. DPN has an essential contribution to increased morbidity and motility in diabetic individuals $[4,5]$.

In DM, the role of the development of neuropathies becomes unclear, but several factors contribute, such as multifactorial, environmental, lifestyle factors, and genetic predispositions [6]. DPN diagnosis is carried out by taking a clinical history, neurologic test, electrophysiological test, and nerve conduction studies 
by standard electromyography machine. Symptoms include decreased sensation, tingling, and burning sensation of feet, legs, and toes $[7,8]$.

CRP belongs to nonspecific acute-phase protein, produced by liver during inflammation, infection, and tissue damage [9]. It is well accepted that increased levels of CRP and interleukin (IL)- 6 in patients with diabetes are directly related to diabetic Neuropathy [10].

Adenosine deaminase (ADA) is the hydrolytic enzyme of adenosine and widely distributed in various tissues [11]. ADA is an essential enzyme for regulating the bioactivity of insulin [12]. The anti-inflammatory effect is found to exert adenosine, and therefore the inflammatory response is regulated by ADA [13]. The increased ADA level indicates the increased insulin resistance and immuno-pathogenesis of type 2 diabetes mellitus [14].

The purpose of the study was to evaluate the serum levels of ADA and CRP in diabetic patients with and without peripheral neuropathy.

\section{Material and methods}

We conducted a cross-sectional study on diabetic patients with and without neuropathy attending the general medicine department of Kasturba Hospital, Manipal, Karnataka, India, for a routine check-up from December 2019 to March 2020. After obtaining IEC approval (IEC No. 261/2019) and registering for Clinical Trial Registration India (CTRI/2019/06/019911), patients who were willing to provide written informed consent were recruited for the study. A detailed clinical history along with fasting blood glucose levels were collected. All patients were screened for neuropathy. Neuropathy testing was done using Michigan's Neuropathy Screening Instrument (MNSI), vibration perception threshold (VPT) by biothesiometer and $10 \mathrm{~g}$ Semmes Weinstein monofilament test. MNSI contains two separate assessments to assess distal symmetrical DPN. First part has self-administrated questionnaire and second part includes assessment of lower extremity by foot examination, angle reflexes, vibration sensation. Large fiber dysfunction was assessed by VPT and loss of protective sensation by monofilament test. Study participants were divided into two groups based on the presence or absence of DPN. All patients aged between 30 and 70 years diagnosed with DM were included. Diabetes mellitus with other neurological dysfunction and hypertension, liver, kidney or thyroid disease, rheumatoid arthritis, pyogenic infections, or autoimmune disorders were excluded from the study to avoid confounding variables.

\section{Sample size}

Taking a standard deviation of 13.56 from the previous study and six units as the clinically signifi- cant difference at $5 \%$ level of significance and $80 \%$ power, the sample size required was 80 per group. We included 160 patients in the study, 80 in each group DPN (48 males, 52 females), and DM (42 males, 32 females).

\section{Estimation of biomarkers}

Leftover serum samples were collected from the clinical biochemistry laboratory and stored at $-20^{\circ} \mathrm{C}$ until the analysis of markers. The biomarker levels in each serum sample were estimated using commercial reagent kits. Serum CRP levels were determined using the ELISA kit (Calbiotech). The CRP ELISA kit is a solid phase direct sandwich method. The diluted samples and conjugate reagent (anti-CRP Biotin \& HRP) were added to the wells coated with Streptavidin. CRP in the patient sample forms a sandwich between two specific antibodies to CRP. Unbound protein and HRP conjugate washed off by wash buffer. Upon the addition of TMB substrate, the intensity of the color is proportional to the concentration of CRP in the samples.

Serum ADA was estimated using the spectrometry method (Agapee kit). The ADA assay was based on the enzymatic deamination of adenosine to ionize, which is converted to hypoxanthine by purine nucleoside phosphorylase (PNP), which is further converted to uric acid and hydrogen peroxide $\left(\mathrm{H}_{2} \mathrm{O}_{2}\right)$ by xanthine oxidase (XOD). Hydrogen peroxide is further reacted with n-Ethyl-N-(2-hydroxy-3-sulfopropyl)-3-methylaniline and 4- amino antipyrine in the presence of peroxidase to generate a quinone dye which was monitored in a kinetic manner.

The value of FBG, PPBS, $\mathrm{HbA} 1 \mathrm{C}$, urea, creatinine, total cholesterol, triglycerides, $\mathrm{LDL}$, and $\mathrm{HDL}$ was collected prospectively from patient medical records.

\section{Result}

A total of 160 patients were included in the study, of which 80 were with DPN. The mean age was $61 \pm$ 9.2 years in the DPN group (Table 1 ) and $53.87 \pm 8.84$ years in the Diabetic group $(P=<0.001)$ (Table 1). The majority of the patients had an age within the range of 51-60 years. (Fig 1.)

The mean duration of diabetes was $5.9 \pm 4.7$ years and DPN $2.1 \pm 1.5$ years (Table 1 ). The comparison of demographic data showed a significant difference in age and duration of diabetes.

\section{Comparison of serum CRP and ADA in diabetic patients with and without DN \\ When data was analyzed, a significant increase in the ADA level was seen in the DPN group $(P=0.032)$.}


Table 1. Comparison of demographic features

\begin{tabular}{lccc}
\hline Variable & DPN (80) & Diabetic (80) & P value \\
\hline Age (years) & $61 \pm 9.2$ & $53.87 \pm 8.84$ & $<0.001$ \\
Duration in years & $2.1 \pm 1.5$ & $5.9 \pm 4.7$ & $<0.001$ \\
Gender (M/F) & $(1.6) 48 / 32$ & $(1.1) 42 / 38$ & 0.412 \\
Fasting blood glucose [mg/dL] & $175.37 \pm 79.80$ & $154.32 \pm 45.83$ & 0.207 \\
\hline
\end{tabular}

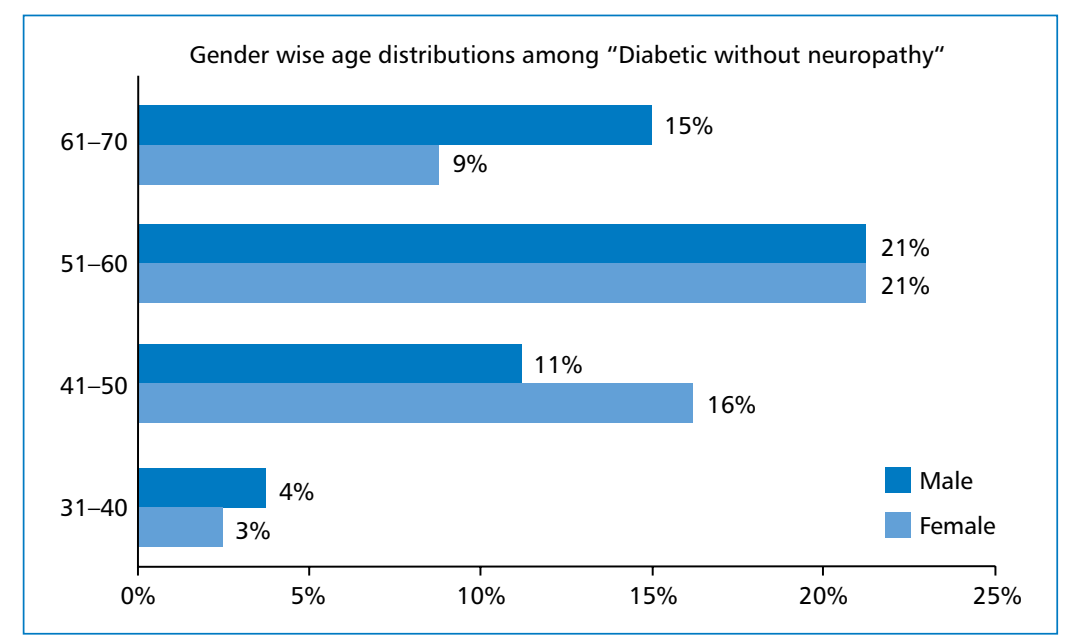

Figure 1. Bar graph showing that $21 \%$ of male and female patients in the age group of 51-60 years were diabetic without neuropathy, whereas only $3 \%$ among females in the age group of 31-40 years were found diabetic without neuropathy

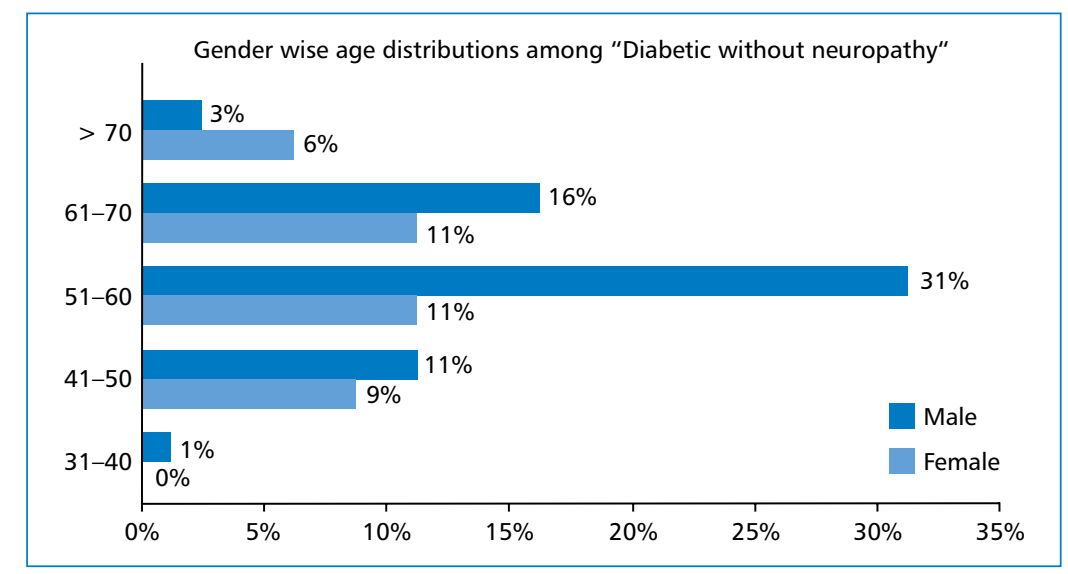

Figure 2. Bar graph showing that $31 \%$ of males in the age group of $51-60$ years were diabetic with neuropathy, whereas only $1 \%$ among the age group of 31-40 years were diabetic with neuropathy. In the elderly population (61-70 and above), there were more cases of diabetic neuropathy

The mean ADA level was 21.70 in patients with DPN. The difference in blood urea values between the groups was significant $(P=0.002)$. No significant difference was seen in the CRP and creatinine levels between two groups $(P=0.123)(P=0.06)$ (Table 2$)$.

\section{The proportion of high ADA and CRP} levels in patients with diabetic neuropathy

In diabetic patients without neuropathy, 24 out of 80 patients had a high level of ADA, and 6 patients had a high CRP level. 
Table 2. Comparison of biochemical parameters in diabetic patients with and without DPN

\begin{tabular}{lccc}
\hline Variable & DPN & Diabetic & P value \\
\hline ADA & $21.70 \pm 28.77$ & $20.75 \pm 17.15$ & 0.032 \\
CRP & $3.89 \pm 5.48$ & $4.19 \pm 2.67$ & 0.123 \\
Urea & $19.84 \pm 6.34$ & $26 \pm 7.15$ & 0.002 \\
\hline
\end{tabular}

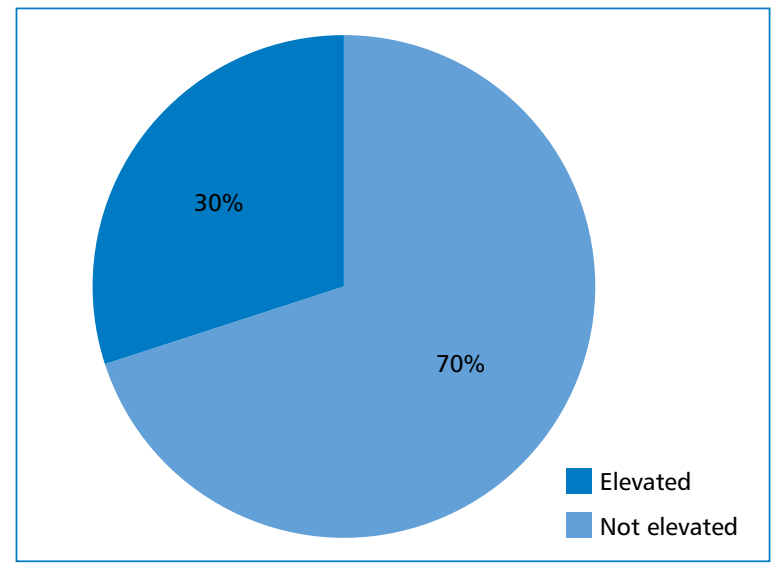

Figure 3. Elevated levels of ADA in DM

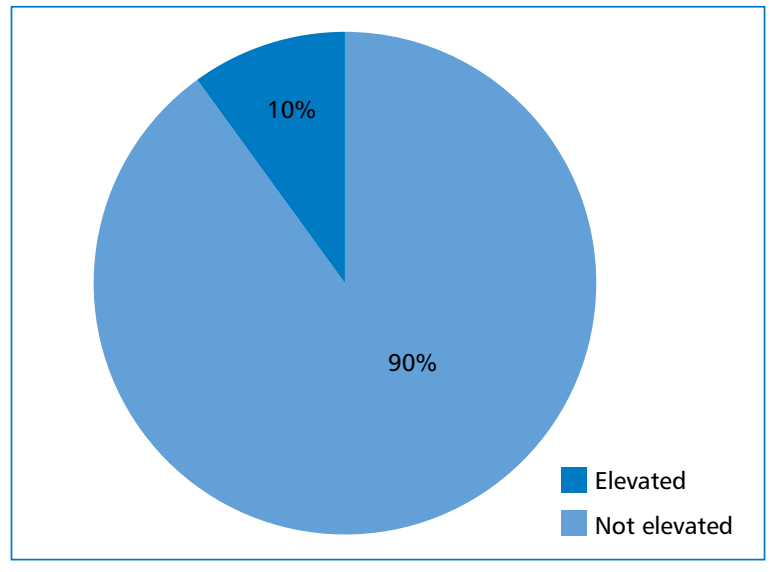

Figure 4. Elevated levels of CRP in DM

In DPN, 28 out of 80 patients had a high level of ADA, and 8 patients had a high level of CRP.

\section{Discussion}

This study was an attempt to compare and determine the proportion of serum ADA and CRP levels in diabetic patients with and without Neuropathy, which may be useful for screening DPN. We observed a significantly increased level of serum ADA in patients with DPN compared to non-DPN patients. There is a lack of literature that can show ADA can be used as a biomarker for DPN.

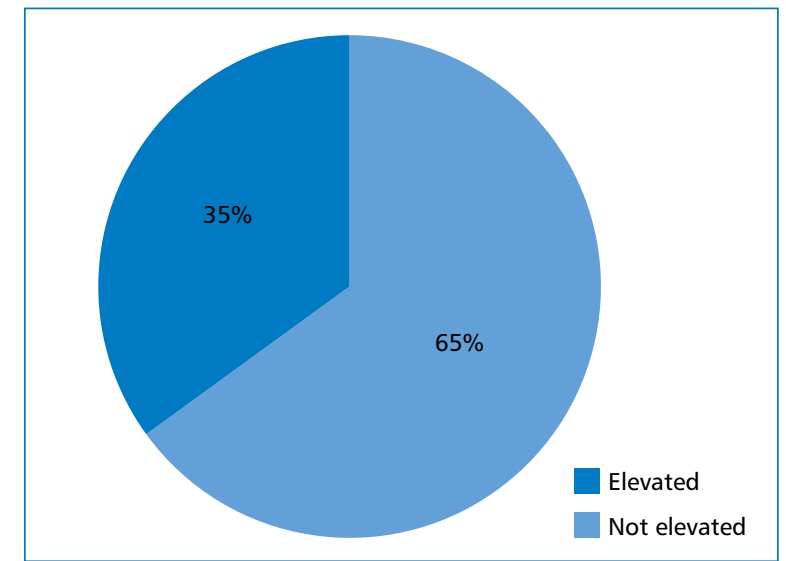

Figure 5. Elevated levels of ADA in DPN

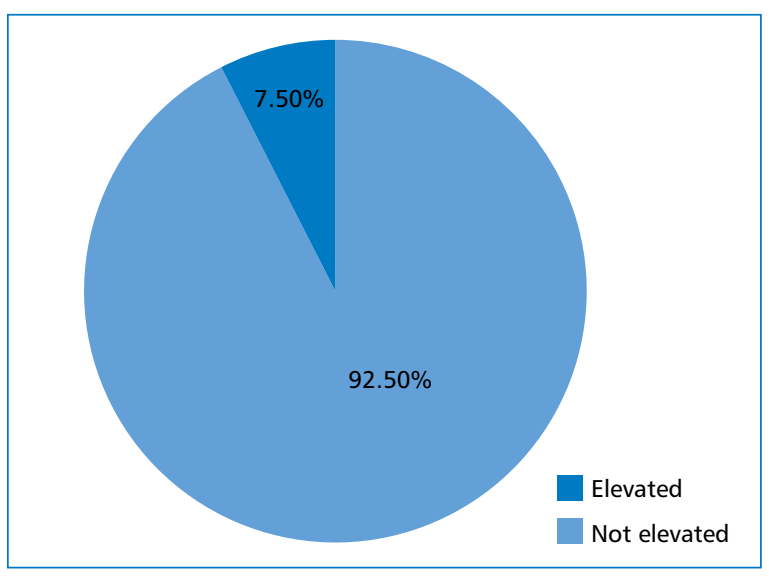

Figure 6. Elevated levels of CRP in DPN

The comparison of demographic data showed a significant difference in age and duration of diabetes. In the present study, the proportion of male and female are equal in diabetic patients without neuropathy, and the ratio of males was higher in patients with diabetic neuropathy. The difference in blood urea values between the DPN and non-DPN groups was significant, and no significant difference was seen in CRP and creatinine levels between the two groups. Levels of CRP were significantly correlated with increased odds of DPN. Additionally, our study also examined the proportion of CRP and ADA in both groups and revealed that 
compared to the CRP level, the ADA level was slightly higher in the DPN group.

Diabetic neuropathy is associated with several cellular and systemic processes that manifest as inflammatory dysfunction. We observed significantly high ADA being a marker of inflammation in DPN patients.

As the low-grade inflammation is a contributing factor of diabetic neuropathy, the current research targets inflammation that may alleviate diabetic neuropathy [15]. In this study, we noticed an elevated serum ADA levels in most patients with diabetic neuropathy, which could provide a better diagnosis and further treatment of neuropathy.

\section{Conclusion}

High ADA level may be a warning signal of peripheral neuropathy in diabetic patients. This study demonstrates that ADA is correlated with DPN's presence; this could be used as an essential indicator for the investigation of DPN. Further research with a greater sample size should be performed to generalize these results.

\section{Conflict of interest}

The authors declare no conflicts of interest.

\section{Acknowledgment}

The authors would like to thank all the patients who participated in the study and also are grateful to the Department of Biochemistry, Kasturba Medical College, MAHE, Manipal, for the support in sample collection.

\section{REFERENCES}

1. Yuen L, Saeedi $P$, Riaz M, et al. Projections of the prevalence of hyperglycaemia in pregnancy in 2019 and beyond: Results from the International Diabetes Federation Diabetes Atlas, 9th edition. Diabetes Res Clin Pract. 2019; 157: 107841, doi: 10.1016/j. diabres.2019.107841, indexed in Pubmed: 31518656.

2. Patterson CC, Karuranga S, Salpea P, et al. Worldwide estimates of incidence, prevalence and mortality of type 1 diabetes in children and adolescents: Results from the International Diabetes
Federation Diabetes Atlas, 9th edition. Diabetes Res Clin Pract. 2019; 157: 107842, doi: 10.1016/j.diabres.2019.107842, indexed in Pubmed: 31518658.

3. Jameson JL, Kasper DL, Longo DL, Fauci AS, Hauser SL, Loscalzo J. Harrison's principles of internal medicine. Mcgraw Hill Education, New York Chicago San Francisco 2018.

4. Vinik Al, Nevoret M-L, Casellini C, Parson H. Diabetic Neuropathy. Endocrinology and Metabolism Clinics of North America 2020;42(4):747-787. https://www.sciencedirect.com/science/ article/abs/pii/S0889852913000522?via\%3Dihub (17.05.2020).

5. Sandireddy R, Yerra VG, Areti A, Komirishetty P, Kumar A. Neuroinflammation and Oxidative Stress in Diabetic Neuropathy: Futuristic Strategies Based on These Targets. International Journal of Endocrinology. 2014. https://www.hindawi.com/journals/ ije/2014/674987/ (17.05.2020).

6. M. Wilson N, E. Wright D. Inflammatory Mediators in Diabetic Neuropathy. Journal of Diabetes \& Metabolism 2012 ;01(S5). https://www.longdom.org/open-access/inflammatory-mediatorsin-diabetic-neuropathy-2155-6156.S5-004.pdf (17.05.2020).

7. Song SO, Kim KJ, Lee B-W, Kang ES, Cha BS, Lee HC. Serum glycated albumin predicts the progression of carotid arterial atherosclerosis. Atherosclerosis. 2012;225(2):450-455. https:// www.atherosclerosis-journal.com/article/S0021-9150(12)006181/fulltext (17.05.2020)

8. Xu F, Zhao LH, Su JB, et al. The relationship between glycemic variability and diabetic peripheral neuropathy in type 2 diabetes with well-controlled HbA1c. Diabetol Metab Syndr. 2014; 6(1): 139, doi: 10.1186/1758-5996-6-139, indexed in Pubmed: 25530811.

9. Vigushin DM, Pepys MB, Hawkins PN. Metabolic and scintigraphic studies of radioiodinated human C-reactive protein in health and disease. J Clin Invest. 1993; 91(4): 1351-7.

10. Pepys MB, Hirschfield GM. C-reactive protein: a critical update. Journal of Clinical Investigation. 2003;111(12):1805-12. https:// www.ncbi.nlm.nih.gov/pmc/articles/PMC161431/ (17.05.2020).

11. Franco $R$, Valenzuela $A$, Lluis $C$, et al. Enzymatic and extra enzymatic role of ecto-adenosine deaminase in lymphocytes. Immunological Reviews. 1998 Feb. ; 161(1): 27-42.

12. Hoshino T, Yamada K, Masuoka K, Tsuboi I, Itoh K, Nonaka K, Oizumi K. Elevated adenosine deaminase activity in the serum of patients with diabetes mellitus. Diabetes Research and Clinical Practice. 1994;25(2):97-102. https://www.diabetesresearchclinicalpractice.com/article/0168-8227(94)90034-5/pdf (17.05.2020).

13. Aruna $S$, Suchitra M, Suresh V. Adenosine deaminase activity in type 2 diabetes mellitus. J Clin Scientific Res. 2017; 6(4): 254.

14. Prakash M, Chennaiah S, Murthy Y, Anjaiah E, Rao A, Suresh C. Altered Adenosine Deaminase Activity in Type 2 Diabetes Mellitus. JIACM. 2006;7(2):114-21. http://medind.nic.in/jac/t06/i2/ jact06i2p114.pdf (17.05.2020)

15. Pop-Busui R, Ang L, Holmes C, et al. Inflammation as a Therapeutic Target for Diabetic Neuropathies. Curr Diab Rep. 2016; 16(3): 29, doi: 10.1007/s11892-016-0727-5, indexed in Pubmed: 26897744. 